\begin{tabular}{ccc}
\hline AECORDS OF PHARMACEUTICAL \\
AND BIOMEDICAL SCIENCES
\end{tabular}

\title{
Shotgun Metagenomic Analysis of Cutaneous Microbiome in Patients with Atopic Dermatitis
}

\author{
Mohammed Ramadan a , Samar Solyman ${ }^{\text {b }}$, Mamdouh Yones ${ }^{\text {a }}$, Hamada Halaby ${ }^{\text {a }}$, \\ Yasser Abdallah ${ }^{c}$, Amro Hanora ${ }^{b^{*}}$ \\ ${ }^{a}$ Department of Microbiology and Immunology, Faculty of Pharmacy, Al-Azhar University, Assiut, Egypt, \\ ${ }^{b}$ Department of Microbiology and Immunology, Faculty of Pharmacy, Suez Canal University. Ismaillia, \\ Egypt, ' Department of Dermatology, Faculty of medicine, Al-Azhar University, Assiut, Egypt.
}

Received on: 01.01. 2019

Revised on: 21. 01. 2019

Accepted on: 30. 01. 2019

Correspondence Author:

Tel:+201000323406.

E-mail address:

ahanora@yahoo.com

\begin{abstract}
Atopic dermatitis $(\mathrm{AD})$ is a chronic complex inflammatory skin disorder. $\mathrm{AD}$ characterized by multiple contributing factors, such as impairment in skin barrier integrity, participation of either innate or adaptive immunity arms, in addition to the pivotal roles of cutaneous microbiome. The analysis of cutaneous microbiota was performed using shotgun metagenomic sequencing on an Illumina HiSeq platform in parallel with 16s rRNA genes using MiSeq sequencing. Two samples for each sequencing technique were collected from one child and one adult subjects with AD. Metagenomic data from 16srRNA was in accordance with those from shotgun sequencing. Taxonomic profile of samples at genus level showed overrepresentation of Streptococcus in child and Staphylococcus in adults. Species level analysis of reads revealed monoclonality of Staphylococcus aureus community in comparison to heterogeneity of Staphylococcus epidermidis. 16S rRNA gene based analysis was unable to provide comprehensive description of bacterial communities, while shotgun metagenomic analyses described the composition of microbiota with high resolution at different taxonomic levels especially subspecies and single nucleotide variants levels. Finally, further studies using shotgun metagenomic approaches will be required for characterization of microbiome in healthy and diseased subjects.
\end{abstract}

Keywords: Doping, Atopic dermatitis, Shotgun metagenomic analysis, Microbiome, 16srRNA.

\section{Introduction}

Atopic dermatitis is an allergic disease that impacts the life of about $20 \%$ of children and $2.3-4.9 \%$ between adults worldwide (Barbarot et al., 2018). Understanding the role of cutaneous microbiota in development as well as worsening of disease severity is essential for developing new treatment polices for elimination and prophylaxis against $\mathrm{AD}$ (Nakatsuji and Gallo, 2018).
Although discovery and exploration of human microbiome mainly depend on annotation of $16 \mathrm{~S}$ rRNA hypervariable regions, these approaches in addition to traditional culture based unable to provide full description of underlying microbial communities (Zhang et al., 2011) . On the other hand, shotgun metagenomic tools enable imaging of entire members of human associated microbial communities including bacteria, fungi and viruses (Ranjan et al., 2016). The current study was 
launched to identify the association between the composition of bacterial communities and $\mathrm{AD}$ in Egyptian patients.

\section{Results}

\subsection{Taxonomic profiling of cutaneous microbiota}

Bioinformatics sequence processing and annotation of 16s rRNA reads from two sequencing approaches were in agreement to each other at higher taxonomic levels from phylum to genus levels (Fig. $1)$.

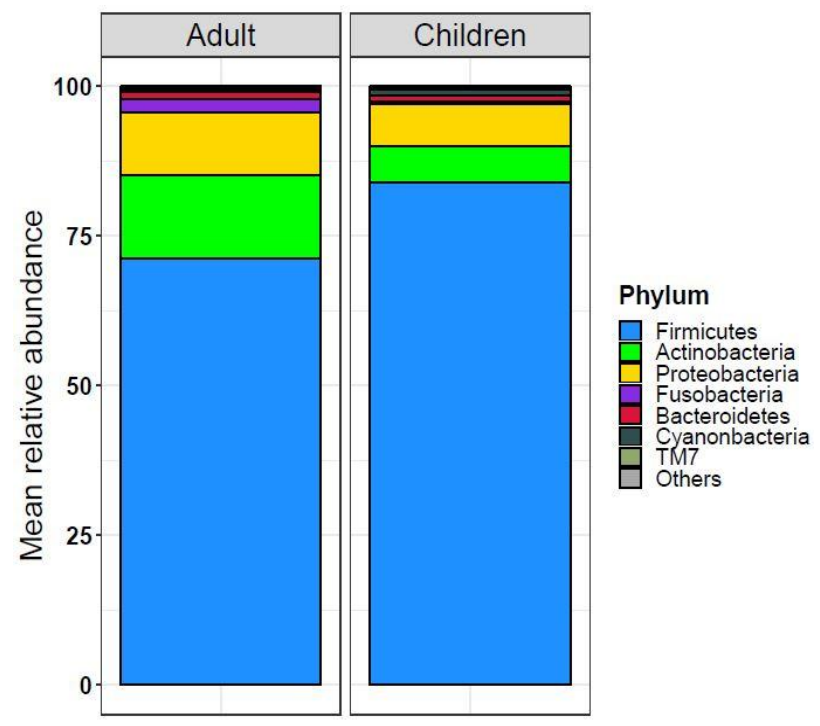

Figure 1. Mean relative abundance of most predominant phylum in cutaneous microbiota in patients with AD

At genus level, genera belonging to Firmicutes included Staphylococcus, Streptococcus and Bacillus, were detected with comparable abundance where Staphylococcus was enriched in both children and adults but with overrepresentation in adults. On the other hand Streptococcus was more significantly abundant in children compared to adults. Moreover, the representation of Cutibacterium and Corynebacterium significantly enriched in adults and diminished in children.

\subsection{Species level and single nucleotide variant annotation of sequencing reads}

Staphylococcus species, especially $S$. hominis and $S$. haemoliticus were assigned to the same clade. As compared to 16SrRNA sequencing, taxonomic assignment of assembled shotgun sequencing gets rid of misclassification of unassigned sequences where Staphylococcus species were classified to $S$, aureus, S. epidermidis, S. haemoliticus, S. cohnii, S. aureus, S. caprae and S. hominis (Fig. 2). Although Sequences assigned to $S$. aureus were annotated to 16 separate clades, $S$. aureus community in adult was predominant by single clade while children were predominant by another clade.

\subsection{Nonbacterial inhabitants on skin associated with AD}

Nonbacterial members of skin microbial communities included fungi and viruses that were detected with lower density compared to bacterial residents. Viral community was composed of predominate phages with few viruses. On the other hand fungal residents mainly represented by Malasseziaceae family that dominated by $M$. globosa and $M$. restricta, in addition to Aspergillaceae family.

\section{Discussion}

$\mathrm{AD}$ is a multifaced skin disorder with increased prevalence over the world including developed industrialized countries as well as developing countries (Ng et al., 2018, Goh et al., 2018). Deep understanding of cutaneous microbiome using advanced tools seems to provide a better explanation of the potential contributions of $\mathrm{AD}$ associated microbial communities in the pathogenesis of AD (Chng et al., 2016).

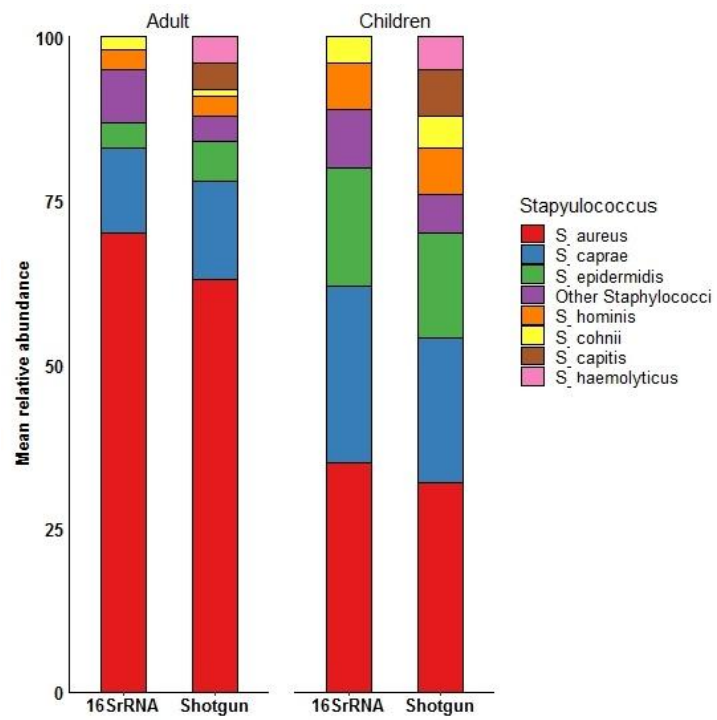

Figure 2. Mean relative abundances of Staphylococcus species in skin microbiota using 16S rRNA based sequencing and shotgun metagenomic analysis. 
Age dependent shifts in skin microbiota previously reported and showed distinct age specific patterns in skin microbiota (Oh et al., 2012). Composition of microbiome in children and adults at higher taxonomic levels showed predominance of four major phyla Firmicutes, Proteobacteria, Actinobacteria and Bacteroidetes that previously reports with different rank where Proteobacteria was detected with lowered abundance, while Cutibacterium were found with increased proportions with progress in age as well as inverse correlation with Staphylococcus could be linked to maturation related physiological and endocrinologic changes (Wang et al., 2008, Abrahamsson et al., 2012, Kong et al., 2012).

S. aureus was detected with enriched abundance in adult and children. These findings are consistent with previous reports that defined $S$. aureus as a major player in pathogenesis of AD (Williams and Gallo, 2015, Totte et al., 2016).

In this study, $M$. dermatis and $M$. sympodalis were detected with enriched abundances in adult and child respectively which could be contributed to species switching in pathogenesis of $\mathrm{AD}$ (Han et al., 2018). The current study provides new insight about microbiota associated to AD in Egyptian patients using shotgun metagenomic approach.

\section{References}

Abrahamsson T.R., Jakobsson H.E., Andersson A.F., Bjorksten B., Engstrand L.Jenmalm M.C., 2012. Low diversity of the gut microbiota in infants with atopic eczema. J Allergy Clin Immunol, 129, 434-40, 440.e1-2.

Barbarot S., Auziere S., Gadkari A., et al., 2018. Epidemiology of atopic dermatitis in adults: Results from an international survey. Allergy, 73, 12841293.

Chng K.R., Tay A.S.L., Li C., et al., 2016. Whole metagenome profiling reveals skin microbiomedependent susceptibility to atopic dermatitis flare. Nature Microbiology, 1, 16106.

Goh Y.Y., Keshavarzi F.Chew Y.L., 2018. Prevalence of Atopic Dermatitis and Pattern of Drug Therapy in Malaysian Children. Dermatitis, $29,151-161$.
Han S.H., Cheon H.I., Hur M.S., et al., 2018. Analysis of the skin mycobiome in adult patients with atopic dermatitis. Experimental Dermatology, 27, 366-373.

Kong H.H., Oh J., Deming C., et al., 2012. Temporal shifts in the skin microbiome associated with disease flares and treatment in children with atopic dermatitis. Genome Res, 22, 850-9.

Nakatsuji T.Gallo R.L., 2018. The role of the skin microbiome in atopic dermatitis. Annals of Allergy, Asthma \& Immunology.

Ng M.S., Tan S., Chan N.H., Foong A.Y.Koh M.J., 2018. Effect of atopic dermatitis on quality of life and its psychosocial impact in Asian adolescents. Australas J Dermatol, 59, e114-e117.

Oh J., Conlan S., Polley E.C., Segre J.A.Kong H.H., 2012. Shifts in human skin and nares microbiota of healthy children and adults. Genome Med, 4, 77.

Ranjan R., Rani A., Metwally A., Mcgee H.S.Perkins D.L., 2016. Analysis of the microbiome: Advantages of whole genome shotgun versus $16 \mathrm{~S}$ amplicon sequencing. Biochemical and Biophysical Research Communications, 469, 967 977.

Totte J.E., Van Der Feltz W.T., Hennekam M., Van Belkum A., Van Zuuren E.J.Pasmans S.G., 2016. Prevalence and odds of Staphylococcus aureus carriage in atopic dermatitis: a systematic review and meta-analysis. Br J Dermatol, 175, 687-95.

Wang M., Karlsson C., Olsson C., et al., 2008. Reduced diversity in the early fecal microbiota of infants with atopic eczema. J Allergy Clin Immunol, 121, 129-34.

Williams M.R.Gallo R.L., 2015. The role of the skin microbiome in atopic dermatitis. Curr Allergy Asthma Rep, 15, 65.

Zhang J., Chiodini R., Badr A.Zhang G., 2011. The impact of next-generation sequencing on genomics. J Genet Genomics, 38, 95-109. 